\title{
Aerobic exercise reduces triglycerides by targeting apolipoprotein C3 in patients with coronary heart disease
}

\author{
Yating Wang | Li Shen | Danyan Xu $\odot$
}

Department of Cardiovascular Medicine, The Second Xiangya Hospital, Central South University, Changsha, China

\section{Correspondence}

Danyan Xu, PhD, MD, Department of Cardiovascular Medicine, The Second Xiangya Hospital, Central South University, Changsha, Hunan 410011, China.

Email: xudanyan02@csu.edu.cn

Funding information

National Nature Scientific Funding of China, Grant/Award Number: 81672264, 81702240; the Fundamental Research Funds for the Central Universities of Central South University, Grant/Award Number: 2018zzts 260
Background: Aerobic exercise, which has been shown to have beneficial effects on plasma lipids, has been recommended as an effective measure to improve the prognosis of individuals with coronary heart disease (CHD). Apolipoprotein C3 (apoC3) is associated with hypertriglyceridemia and is therefore closely related to CHD.

Hypothesis: We measured apoC 3 concentration change in patients with $\mathrm{CHD}$ before and after long-term aerobic exercise.

Methods: Thirty-eight patients with coronary heart disease were randomly assigned to a nonexercise group (19 patients) or exercise group (19 patients). Both groups received essential drugs for CHD. The non-exercise group was kept sedentary while the exercise group performed moderate-intensive aerobic exercise for 8 weeks. Lipid levels and apoC3 levels were measured on the first day and 8 weeks later.

Results: Exercise for 8 weeks led to a significant decrease in concentration of triglyceride and apoC3 compared with the baseline. Triglyceride concentration changes were positively associated with apoC3 level changes.

Conclusions: Aerobic exercise can improve the lipid profile. It is effective in decreasing triglycerides by targeting apoC3 levels in patients with coronary heart disease.

\section{KEYWORDS}

aerobic exercise, apolipoprotein C3, atherosclerotic cardiovascular disease, coronary heart disease, triglyceride

\section{1 | INTRODUCTION}

Coronary heart disease (CHD) is the leading cause of mortality worldwide and the major risk factors are sub-optimal levels of lipid and lipoprotein levels. It was previously thought that low-density lipoprotein cholesterol ( $L D L-C)$, rather than triglyceride, was the crucial cause that contributes to $\mathrm{CHD} .{ }^{1}$ However, renewed interest in triglyceride levels has generated epidemiological and genetic evidence, which suggests that triglycerides and triglyceride-rich lipoproteins are an additional cause of CHD and all-cause mortality. Recent randomized studies ${ }^{2,3}$ indicated that hypertriglycerides, whether fasting or non-fasting, are closely related to CHD. Researchers showed that triglyceride at 2 to $10 \mathrm{mmol} / \mathrm{L}$ can diffuse into the arterial wall and accumulate, causing

Abbreviations: apoC3, apolipoprotein C3; BMI, body mass index; CHD, coronary heart disease; HDL-C, High-density lipoprotein cholesterol; HL, Hepatic lipase; LDL-C, Low-density lipoprotein cholesterol. atherosclerosis and eventually leading to $\mathrm{CHD} .^{4}$ Further studies showed that patients with established coronary disease may be at risk for recurrence of cardiac events with moderate-to-high elevations in triglyceride levels. ${ }^{5-7}$ Hypertriglycerides are also independent predictors of CHD, as shown by the causal relationships between high triglyceride level and CHD even in individuals with low LDL-C levels after treatment with statins. ${ }^{8}$ As a result, therapeutic methods to reduce hypertriglyceride levels may help to reduce $\mathrm{CHD}$ risk.

Apolipoprotein C3 (apoC3) is a small (8.8 kDa) apolipoprotein composed of 79 amino acid residues. Mainly secreted by the liver and intestine, apoC3 is the most abundant in the apolipoprotein $\mathrm{C}$ family. ApoC3 is closely associated with triglyceride metabolism and is a key regulator of fasting and non-fasting triglyceride levels. ${ }^{9} \mathrm{CHD}$ risk and triglyceride concentration in apoC3 gene mutation carriers were both up to $30 \%$ to $40 \%$ lower than in non-carriers. ${ }^{10}$ Using ISIS 304801, a second-generation antisense inhibitor of apoC3 synthesis, triglyceride levels were lower in hypertriglyceridemia patients with various 
triglyceride baselines. ${ }^{11}$ All these results suggested that apoC3 is a key triglyceride-modulating factor. Therefore, apoC3 is a promising target for lowering high triglyceride levels.

Aerobic exercise has been recommended as an effective measure to improve the prognosis of individuals with CHD. Among its benefits is its ability to lower plasma lipid levels. One study ${ }^{12}$ showed that a combination of aerobic exercise and drug treatment improved lipid level better than any single means. However, there are few studies on the effect of aerobic exercise on apoC3. The purpose of this study was thus to investigate the relationship between aerobic exercise and apoC3 in patients with $\mathrm{CHD}$, with the hypothesis that aerobic exercise affects apoC3.

\section{2 | METHODS}

\section{1 | Subjects}

Thirty-eight patients admitted to our hospital between March and August 2017 were enrolled, and each patient provided informed consent. The protocol conforms to the ethical guidelines of the 1975 Declaration of Helsinki as reflected in a priori approval by the institution's human research committee. All participants were diagnosed with clinical documented CHD through the clinical presentation, ischemic electrocardiographic abnormalities, and coronary angiography findings that showed $\geq 50 \%$ stenosis in at least one main coronary artery with no recent angina attacks. The exclusion criteria for this study were as follows: (a) patients with uncontrolled severe arrhythmia, (b) patients with acute coronary syndrome, (c) patients with severe left ventricle dysfunction or NYHA levels III to IV, (d) patients with musculoskeletal or nervous system disease preventing them from completing the physical exercise assessment and treatment, (e). patients with severe organic disease or electrolyte disturbances, (f) patients with chronic bronchitis or Chronic obstructive pulmonary disease which can affect respiratory function measurement, and (g) patients with severe valvular heart disease, myocarditis, liver or renal function deficiency, cognitive disorder or malignant tumor.

\section{2 | Study design}

Participants were randomly placed into two groups: the non-exercise group (19 patients) and exercise group (19 patients). All patients received essential drugs for coronary heart disease such as antiplatelet drugs, $\beta$-receptor blockers, angiotensin-converting enzyme inhibitors, angiotensin receptor inhibitors or statins; however, no patient received fibrates. The non-exercise group was kept sedentary while the exercise group did moderate-intensive exercise for 8 weeks. Exercise was carried out 5 times per week, and each 45-minutes session involved 15 minutes of warm-up, 25 minutes of exercise and 5 minutes of cooling down. Exercise intensity was controlled at $60 \%$ to $80 \%$ of maximum heart rate. A wearable telemetry Electrocardiography (ECG) monitor (WelchAllyn, New York, New York) was used to monitor heart rate to ensure patient safety, as well as to ensure the target heart rate was reached during the session. All exercise sessions were carried out in the hospital and under professional guidance.
During the exercise period, television programs were provided to the control group. Outside the sessions, physical activity level and food intake in all patients were the same as before enrollment.

\subsection{Clinical and biochemical measurements}

Patient clinical characteristics were recorded, including gender, age, body mass index (BMI) and history of hypertension, diabetes, and smoking. The anthropometric measurements (weight, height, and BMI) were assessed after overnight fasting for at least 10 hours. On the first day and at the end of the 8 weeks, peripheral blood samples were collected in the early morning in the fasting state. Plasma high-density lipoprotein cholesterol (HDL-C), LDL-C, and triglyceride concentrations were measured on a Hitachi 7170A analyzer (Tokyo, Japan) by a specialist in our department who was unaware of the study allocation. Serum sample aliquots were stored in a freezer at $-80^{\circ} \mathrm{C}$.

\section{4 | Measurement of serum ApoC3}

Immediately after collection, blood samples were centrifuged at $3000 \mathrm{rpm}$ for 10 minutes. Then the upper serum samples were collected and stored at $-80^{\circ} \mathrm{C}$ until analysis. ApoC 3 was measured using an apoC3 enzyme-linked immunosorbent assay kit (Abcam Cambridge, UK).

\section{5 | Statistical analysis}

Data were analyzed using Statistical Package for Social Sciences version 21.0. Plots were created with GraphPad Prism version 6.0 (GraphPad Software, Inc., La Jolla, California). Clinical data are presented as mean $\pm \mathrm{SD}$. Comparisons between categorical data were performed with $\chi^{2}$ tests. The baseline levels of the two groups were compared using an independent $t$ test, while comparisons of continuous variables before and after exercise were assessed by paired $t$ test. Coefficients of correlation $(r)$ were calculated by Pearson correlation analysis. Statistical significance was assumed at $P<0.05$.

\section{3 | RESULTS}

\section{1 | Characteristics of study participants}

All 38 participants completed the research: there were no deaths or serious side effects during the study. Baseline characteristics of the patients are shown in Table 1. There were no significant differences in gender, age, BMI or history of hypertension, diabetes or smoking between the two groups. Similarly, levels of triglyceride, LDL-C, HDL$\mathrm{C}$, and apoC3 were also comparable between the groups at baseline. Cardiac management drugs were the same in both groups and did not alter during the study.

\subsection{Serum lipid concentrations before and after exercise}

Concentrations of triglyceride, LDL-C, HDL-C, and apoC3 were not significantly different at 8 weeks compared to baseline in the 
TABLE 1 Demographic and biochemical baseline characteristics of two groups

\begin{tabular}{|c|c|c|c|}
\hline Baseline characteristics & $\begin{array}{l}\text { Non-exercise } \\
\text { group }(\mathrm{n}=19)\end{array}$ & $\begin{array}{l}\text { Exercise group } \\
(\mathrm{n}=19)\end{array}$ & $P$-value \\
\hline Age (years) & $64.36 \pm 12.01$ & $59.84 \pm 11.57$ & 0.24 \\
\hline Gender (male/female) & $14 / 5$ & $16 / 3$ & 0.43 \\
\hline $\mathrm{BMI}\left(\mathrm{kg} / \mathrm{m}^{2}\right)$ & $24.06 \pm 0.60$ & $24.48 \pm 0.66$ & 0.64 \\
\hline $\begin{array}{l}\text { History of hypertension } \\
\quad(\mathrm{n}, \%)\end{array}$ & $16(84.21)$ & $15(78.95)$ & 0.68 \\
\hline History of diabetes (n, \%) & $6(31.58)$ & $4(21.05)$ & 0.46 \\
\hline History of smoking (n, \%) & $11(57.89)$ & $9(47.37)$ & 0.52 \\
\hline $\mathrm{LDL}-\mathrm{C}$ (mmol/L) & $2.26 \pm 0.12$ & $2.34 \pm 0.13$ & 0.66 \\
\hline $\mathrm{HDL}-\mathrm{C}(\mathrm{mmol} / \mathrm{L})$ & $1.08 \pm 0.05$ & $0.97 \pm 0.04$ & 0.11 \\
\hline Triglyceride (mmol/L) & $2.08 \pm 0.23$ & $1.94 \pm 0.18$ & 0.65 \\
\hline ApoC3 (mg/L) & $1416 \pm 83.31$ & $1381 \pm 67.01$ & 0.75 \\
\hline
\end{tabular}

Abbreviations: ApoC3, apolipoprotein C3;BMI, body mass index; LDL-C low-density lipoprotein cholesterol; HDL-C, high-density lipoprotein cholesterol.

sedentary group. However, the exercise group had significantly smaller concentrations of triglyceride $(P<0.01)$ and apoC3 $(P<0.001)$, as well as a significant increase in HDL-C $(P<0.01)$ after 8 weeks compared to baseline levels. However, there was no change in LDL-C concentration (Figure 1).

Given that change in BMI may lead to changes in triglyceride or apoC3 concentration, BMI levels before and after exercise were also compared (Figure 2). However, there was no significant change in BMl after 8 weeks' exercise $(P=0.32)$.

\section{3 | The correlation between the triglyceride and apoC3 change}

To investigate whether changes in triglyceride concentration were because of changes in apoC3 levels, we analyzed the correlation between the changes in the two values, calculated as post-exercise value minus baseline value. Triglyceride level changes were significantly positively associated with apoC3 level changes $(r=0.5605$ $P=0.0126$, Figure 3).

\section{4 | DISCUSSION}

In the present study, we found that an 8-week exercise regime led to a significant decrease in triglyceride and apoC3 concentration compared with baseline, and significantly higher HDL-C. In addition, triglyceride level changes were positively associated with apoC3 level changes.

Dyslipidemia contributes substantially to increased CHD incidence. More attention has been paid to triglyceride concentration since many studies have shown distinct relationships between hypertriglyceride and $\mathrm{CHD}$ risk. One paper ${ }^{13}$ indicated that $\mathrm{CHD}$ risk increased by $32 \%$ and $76 \%$ in high-triglyceride men and women, respectively. A meta-analysis ${ }^{14}$ showed a $72 \%$ correlation between high triglyceride concentrations and $\mathrm{CHD}$ risk, even with corrected HDL-C levels. These data suggested that lowering triglyceride levels might help reduce CHD risk.
Many means have been suggested to lower triglyceride, and the most approved one may be drugs, including fibrates, such as fenofibrate. However, fibrates can lead to transaminase increase. Novel drug therapies, such as fish oils or niacin also help reduce triglycerides; however, these are not available for most patients due to their cost. Therefore, means for lowering triglyceride levels are still required.

In the present study, we observed that exercise over an 8-week period can effectively reduce triglyceride concentration: this is consistent with previous studies. For example, one study ${ }^{15}$ indicated that proper exercise significantly lowered triglyceride concentration and thereby decreased CHD risk. We found that when patients had a lower baseline level of triglyceride, there was only a slight decrease in triglyceride after exercise. In contrast, when baseline triglyceride level was high, the decrease was significant. So, we can conclude that baseline level may be a crucial factor influencing the effect of exercise on triglyceride response.

ApoC3 plays an important role in triglyceride metabolism. Its physiological function is to reduce triglyceride-rich lipoprotein and remnant uptake at the liver by inhibiting lipoprotein lipase (LPL) and hepatic lipase $(\mathrm{HL})$ activity. ${ }^{16}$ As a result, high apoC3 inhibits $\mathrm{LPL}$ and $\mathrm{HL}$ activity to inhibit triglyceride hydrolysis, which leads to an increase in triglyceride level. Thus, targeting apoC3 is a promising approach to reducing triglyceride concentration. In the present study, we showed that an 8-week period of aerobic exercise could lead to a significant decrease of serum apoC 3 concentration.

It is worth noting that the triglyceride decrease in our study was without BMI loss, which means triglyceride reductions were as a results of directly to the exercise intervention. We hypothesized that exercise-induced apoC3 decrease might increase triglyceride hydrolysis through improving LPL function. Unfortunately, we were unable to detect the concentration of LPL and HL. There did not seem to be a correlation between the degree of decrease in apoC3 and its base value, suggesting that exercise affects apoC3 through LPL- or HLindependent pathways. However, the underlying mechanism needs to be further explored. We analyzed the correlation between changes in triglyceride and apoC3 concentrations. As expected, triglyceride concentration changes were positively associated with apoC3 level changes. This reflected the fact that apoC3 was a key factor in modulating triglyceride and suggested that exercise-induced triglyceride changes may be through the apoC3 pathway.

HDL-C levels saw the highest changes after 8 weeks of exercise. Almost all studies focusing on exercise and HDL-C seem to consistently indicate an increase in HDL-C after exercise. One study ${ }^{11}$ showed that patients treated with an antisense inhibitor of apoC3 (ISIS 304801) had increased mean HDL-C levels. Interestingly, our study showed that exercise could also slightly raise HDL-C concentrations. In addition, apoC3 can inhibit LPL, which also participates in the remodeling of $\mathrm{HDL}-\mathrm{C} .{ }^{17}$ However, the relative contributions of the potential underlying mechanisms have yet to be determined.

Notably, we found that LDL-C level was unaltered after long-term exercise, even though both triglyceride and HDL-C changed. This may be because of variations in patient BMI. Several studies indicated that aerobic exercise alone would not have a significant effect on LDL-C levels, unless the patient's mass also altered during training. Besides, research showed that each kilogram of body mass loss may result in LDL-C loss of $0.8 \mathrm{mg} / \mathrm{dL} .{ }^{18}$ It is well known that exercise can lower 

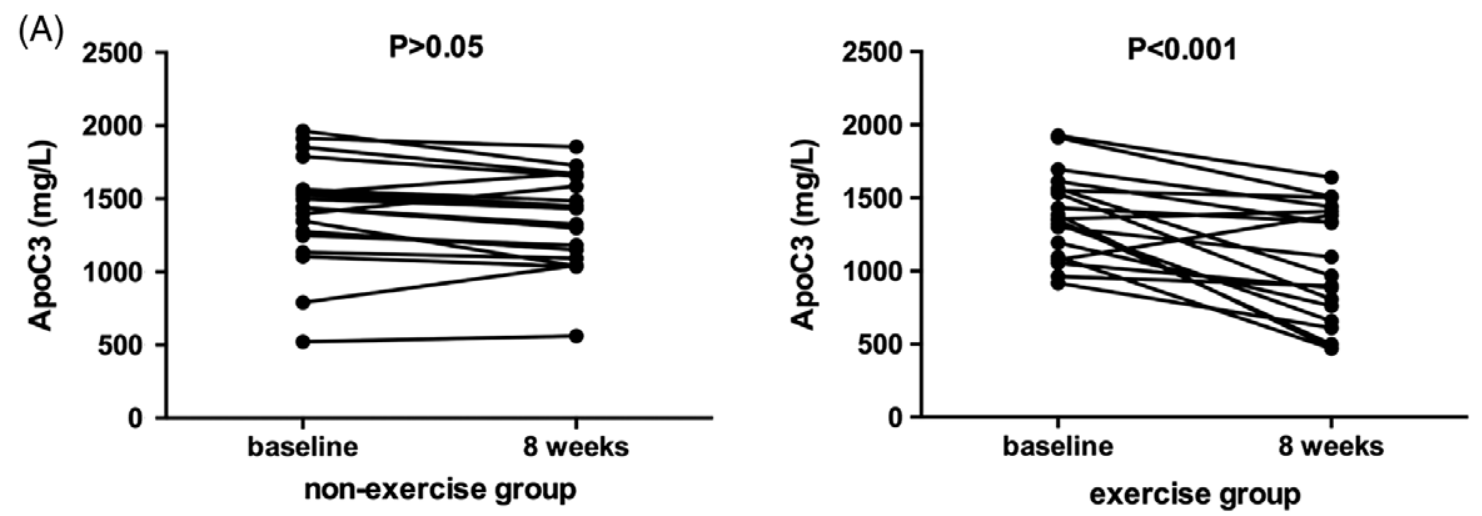

(B)
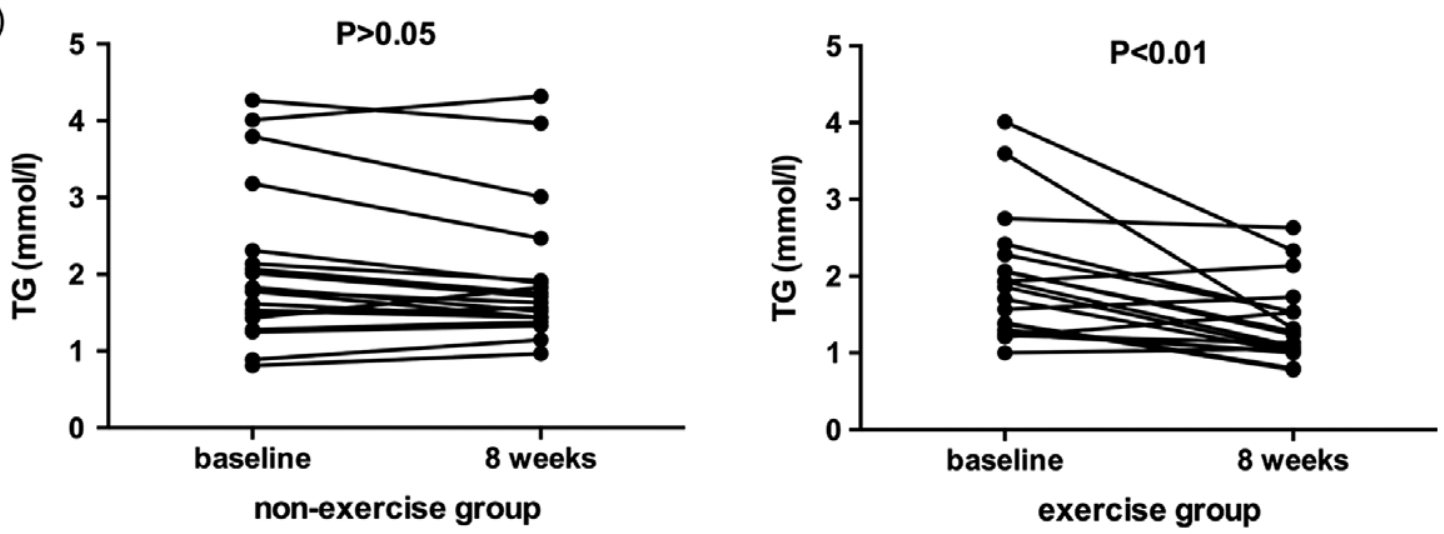

(C)

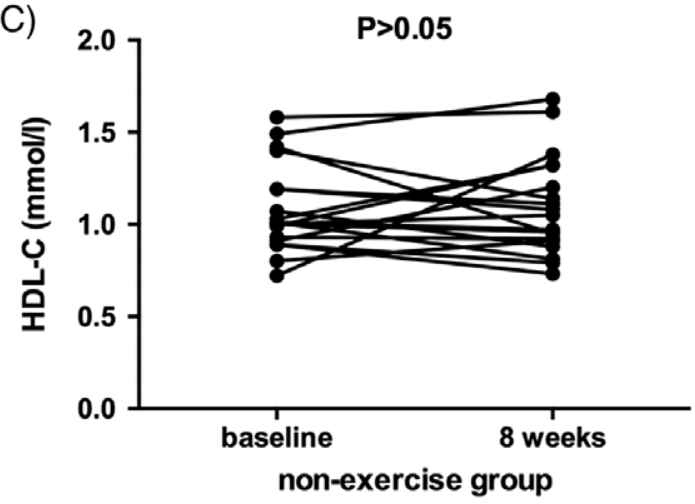

(D)
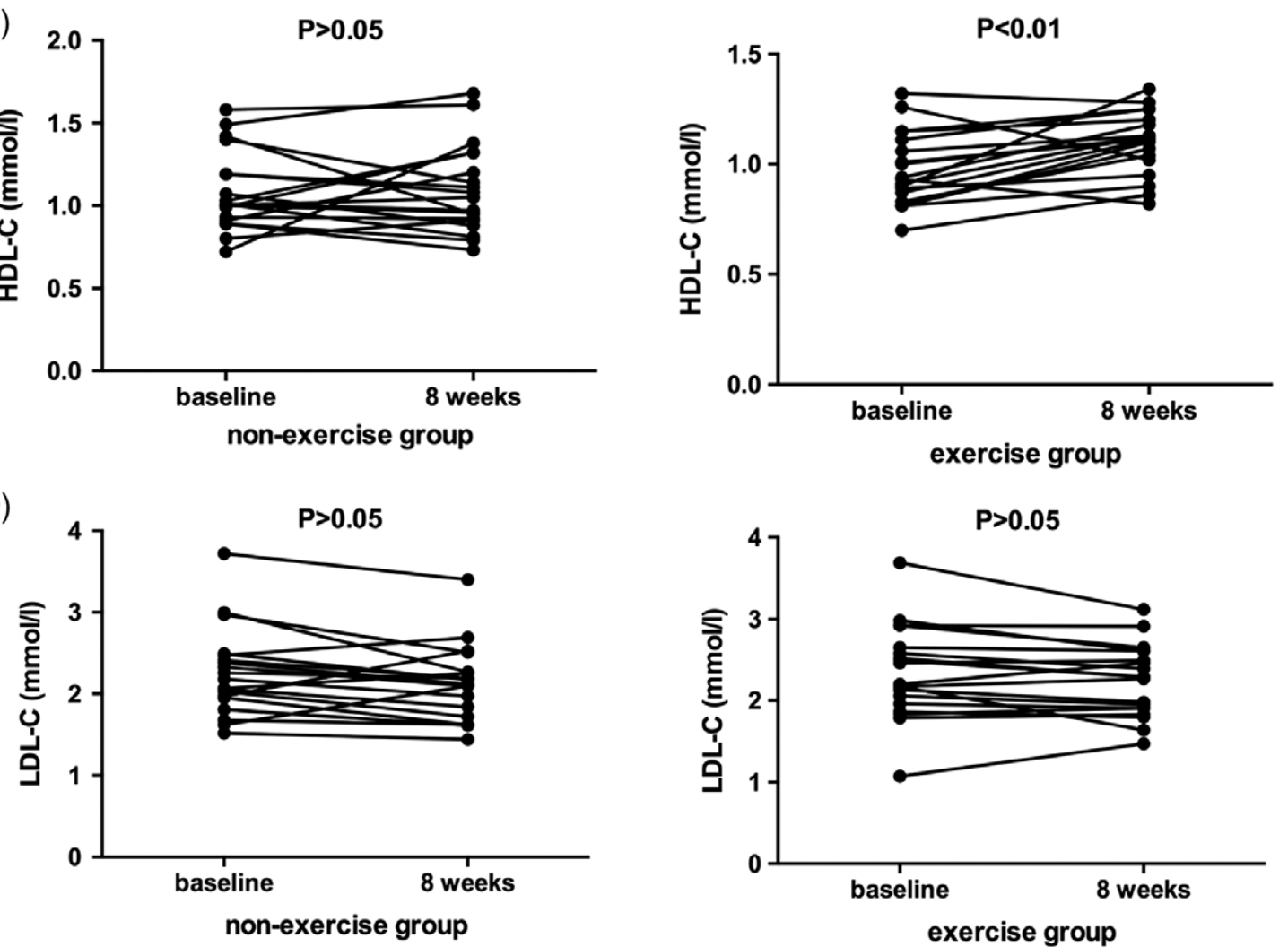

FIGURE 1 Comparison of apolipoprotein C3 (ApoC3), triglyceride, high-density lipoprotein cholesterol (HDL-C), and low-density lipoprotein cholesterol (LDL-C) levels before and after aerobic exercise, paired $t$ test showed that ApoC3 and triglyceride concentrations decreased, HDL-C concentrations increased, and LDL-C concentrations were unaltered after exercise 

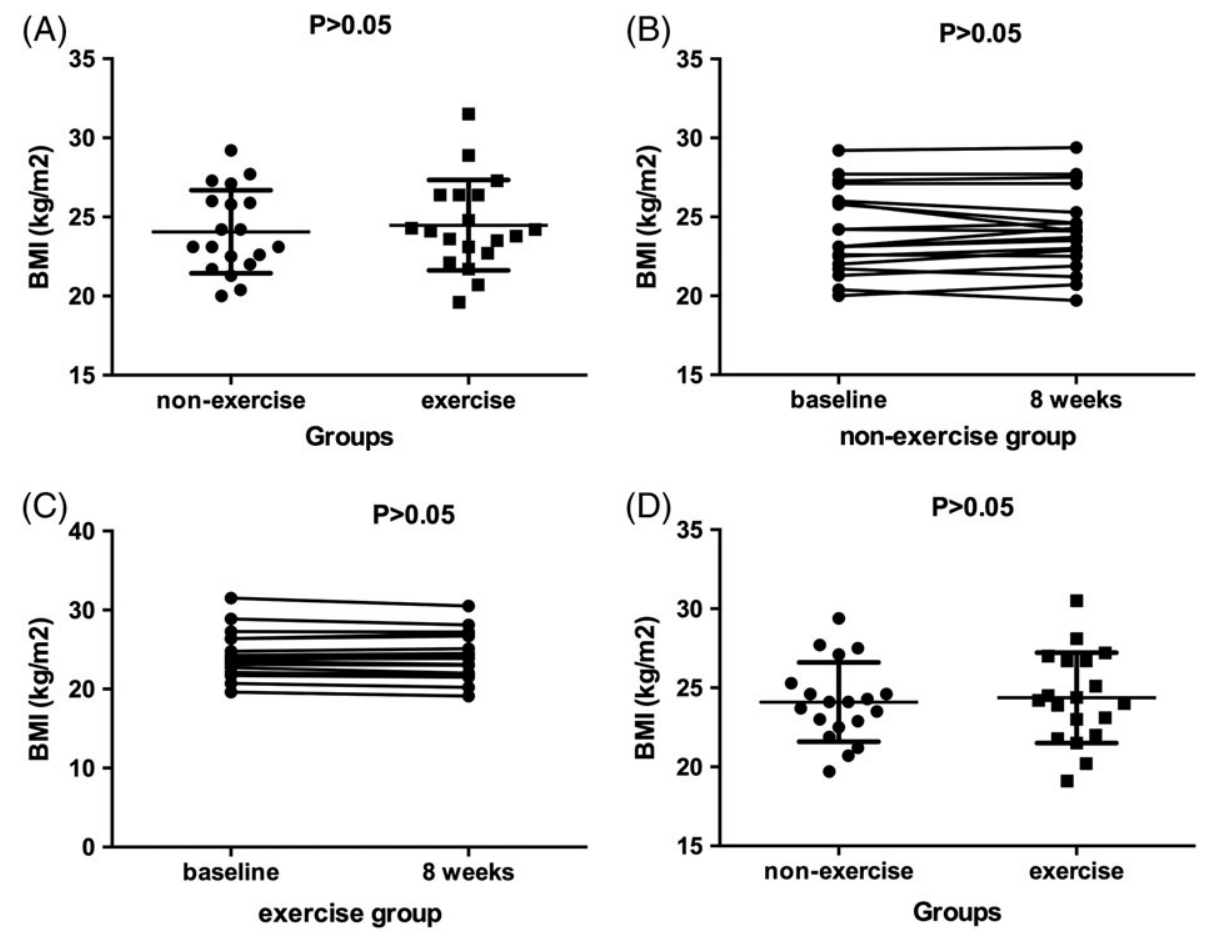

FIGURE 2 A, Comparison of basal body mass index (BMI) levels, unpaired $t$ test showed that there was no difference between non-exercise group and exercise group $\left[(24.06 \pm 0.60) \mathrm{kg} / \mathrm{m}^{2}\right.$ vs $\left.(24.48 \pm 0.66) \mathrm{kg} / \mathrm{m}^{2}, P>0.05\right]$. B, C, Comparison BMI levels before and after the study in two groups, respectively, paired $t$ test showed that BMI levels were unaltered. $\mathrm{D}$, Comparison of BMI levels at the end of the study, unpaired $t$ test showed that there was no difference between non-exercise group and exercise group $\left[(24.09 \pm 0.58) \mathrm{kg} / \mathrm{m}^{2} \mathrm{vs}(24.37 \pm 0.66) \mathrm{kg} / \mathrm{m}^{2}, P>0.05\right]$

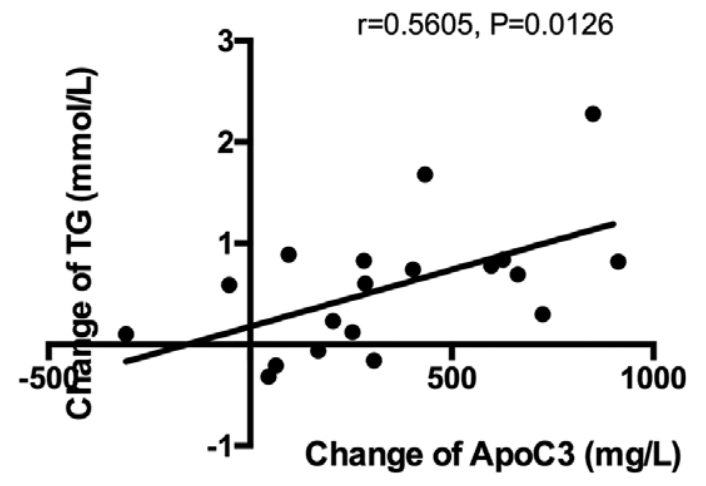

FIGURE 3 The correlation between the triglyceride change (the triglyceride change is equal to its base value minus the value after exercise) and the apolipoprotein C3 (apoC3) change (the apoC3 change is equal to its base value minus the value after exercise) showed that triglyceride concentration changes were positively associated with apoC3 level changes $(r=0.5605, P=0.0126)$

fat mass and elevate lean tissue mass, which may affect the authenticity of the result. However, in our study BMI was unchanged; therefore, it was unlikely that apoC3 levels were influenced by BMI, fat mass, or lean tissue mass.

We acknowledge that the sample size is small. And more researches about mechanisms among apoC3, triglyceride, and exercise are needed.

\section{5 | CONCLUSION}

In conclusion, the results of this study suggest that aerobic exercise could be appropriate for decreasing triglyceride levels in patients with
$\mathrm{CHD}$, and that this may be through an apoC3-regulated mechanism. Also, as aerobic exercise is a cost-effective, non-pharmacological interaction available to the vast majority of the general public, it is worth popularizing. Clinicians should encourage as much physical activity as possible.

\section{ACKNOWLEDGEMENTS}

We thank all subjects who participated in this study, Dr Ming Hu for serum lipids measurements, and the Department of Clinical Laboratory, Second Xiangya Hospital, for their assistance. This work was supported by National Nature Scientific Funding of China (No. 81672264, 81702240), and the Fundamental Research Funds for the Central Universities of Central South University (No. 2018z zts260).

\section{Author contributions}

Y.W. and D.X. designed the study and analyzed the data. All authors contributed intellectually to data interpretation and manuscript preparation.

\section{CONFLICTS OF INTEREST}

No conflict of interest was declared.

\section{ORCID}

Danyan Xu (D) https://orcid.org/0000-0002-8686-5317 


\section{REFERENCES}

1. Sarwar N, Sandhu MS, Ricketts SL, et al. Triglyceride-mediated pathways and coronary disease: collaborative analysis of 101 studies. Lancet. 2010;375(1):634-639.

2. Sarwar N, Perry P, Kaptoge S, et al. Major lipids, apolipoproteins, and risk of vascular disease. JAMA. 2009;302:1993-2000.

3. Freiberg JJ, Tybjaerg-Hansen A, Jensen JS, Nordestgaard BG. Nonfasting triglycerides and risk of ischemic stroke in the general population. JAMA. 2008;300:2142-2152.

4. Nordestgaard BG, Wootton R, Lewis B. Selective retention of VLDL, $\mathrm{HDL}$, and LDL in the arterial intima of genetically hyperlipidemic rabbits in vivo. Molecular size as a determinant of fractional loss from the intima-inner media. Arterioscler Thromb Vasc Biol. 1995;15:534-542.

5. Nordestgaard BG, Varbo A. Triglycerides and cardiovascular disease. Lancet. 2014;384:626-635.

6. Miller M, Stone NJ, Ballantyne C, et al. Triglycerides and cardiovascular disease: a scientific statement from the American Heart Association. Circulation. 2011;123:2292-2333.

7. Chapman MJ, Ginsberg HN, Amarenco P, et al. Triglyceride-rich lipoproteins and high-density lipoprotein cholesterol in patients at high risk of cardiovascular disease: evidence and guidance for management. Eur Heart J. 2011;32:1345-1361.

8. Miller M, Cannon CP, Murphy SA, et al. Impact of triglyceride levels and beyond low-density lipoprotein cholesterol after acute coronary syndrome in the PROVEIT-TIME 22 trial. J Am Coll Cardiol. 2008;51: 724-730.

9. Gaudet D, Brisson D, Tremblay K, et al. Targeting APOC3 in the familial chylomicronemia syndrome. N Engl J Med. 2014;371(2):200-206.

10. Crosby J, Peloso GM, Auer PL, et al. Loss-of-function mutations in APOC3, triglycerides, and coronary disease. N Engl J Med. 2014; 3(371):22-31.

11. Gaudet D, Alexander VJ, Baker BF, et al. Antisense inhibition of apolipoprotein C-III in patients with hypertriglyceridemia. N Engl J Med. 2015;373(5):438-447.
12. Kokkinos PF, Faselis C, Myers J, Panagiotakos D, Doumas M. Interactive effects of fitness and statin treatment on mortality risk in veterans with dyslipidaemia: a cohort study. Lancet. 2013;381:394-399.

13. Austin MA, Hokanson JE, Edwards KL. Hypertriglyceridemia as a cardiovascular risk factor. Am J Cardiol. 1998;81:7B-12B.

14. Sarwar N, Danesh J, Eiriksdottir G, et al. Triglycerides and the risk of coronary heart disease: 10,158 incident cases among 262,525 participants in 29 western prospective studies. Circulation. 2007;115(4): 450-458.

15. Jørgensen AB, Frikke-Schmidt R, Nordestgaard BG, TybjærgHansen A. Loss-of-function mutations in APOC3 and risk of ischemic vascular disease. N Engl J Med. 2014;371:32-41.

16. Norata GD, Tsimikas S, Pirillo A, Catapano AL. Apolipoprotein C-III: from pathophysiology to pharmacology. Trends Pharmacol Sci. 2015; 36:675-687.

17. Brown RJ, Lagor WR, Sankaranaravanan S, et al. Impact of combined deficiency of hepatic lipase and endothelial lipase on the metabolism of both high-density lipoproteins and apolipoprotein B-containing lipoproteins. Circ Res. 2010;107:357-364.

18. Goldberg AC, Hopkins PN, Toth PP, et al. Familial hypercholesterolemia: screening, diagnosis and management of pediatric and adult patients: clinical guidance from the National Lipid Association Expert Panel on familial hypercholesterolemia. J Clin Lipidol. 2011;5:S1-S8.

How to cite this article: Wang Y, Shen L, Xu D. Aerobic exercise reduces triglycerides by targeting apolipoprotein $\mathrm{C} 3$ in patients with coronary heart disease. Clin Cardiol. 2019;42: 56-61. https://doi.org/10.1002/clc.23104 